\title{
Valores de la Economía Social: Gestión de Recursos Humanos y Sostenibilidad
}

\section{Francisco Rincón Roldán, Álvaro López Cabrales}

RESUMEN: Este artículo pretende establecer teóricamente la relación positiva existente entre las entidades de Economía Social y su Sostenibilidad. Nos cuestionamos si son los principios y valores que marcan la creación o fundación de este tipo de entidades las que las hacen más sostenibles, o bien esa relación está mediada por las diferentes prácticas de Gestión de Recursos Humanos que desarrollan estas entidades. Nuestra investigación se desarrolla en el sector de las entidades reguladas por la Ley 5/2011, de 29 de marzo, de Economía Social. En primer lugar, realizaremos una revisión de la literatura para contextualizar el marco actual de la Economía Social, determinando sus principios y valores organizativos más significativos, así como la incidencia de estos en el diseño de ciertas Prácticas de Gestión de Recursos Humanos. Posteriormente estudiaremos una muestra de entidades de Economía Social españolas más representativas, de la cual hemos destacado los tres valores más presentes en ellas, que son concretamente el valor Apoyo Percibido, el valor Respeto y el valor Responsabilidad. Nuestro análisis sugiere que estos tres valores pueden ser importantes para planificar e implementar determinadas Prácticas de Gestión de Recursos Humanos tales como: Selección, Formación y Desarrollo Profesional y, finalmente, la Gestión de la Diversidad. El trabajo termina planteando un modelo teórico y unas proposiciones entre las variables consideradas.

PALABRAS CLAVE: Economía Social, Valores organizativos, Sostenibilidad, Gestión de Recursos Humanos.

CLAVES ECONLIT: A13, E26, 015, Q56.

Cómo citar este artículo/How to cite this article: RINCÓN, F. \& LÓPEZ, A. (2021): "Valores de la Economía Social: Gestión de Recursos Humanos y Sostenibilidad", CIRIEC-España, Revista de Economía Pública, Social y Cooperativa, 102, 33-59. DOI: 10.7203/CIRIEC-E.102.18291.

Correspondencia: Francisco Rincón Roldán, Universidad Pablo de Olavide, fjrinrol@upo.es, ORCID: 0000-0002-1181-9703; Álvaro López Cabrales, Universidad Pablo de Olavide, alopcab@upo. es, ORCID: 0000-0001-6735-0425. 


\section{Expanded abstract}

\section{Social Economy Values: Human Resource Management and Sustainability}

\section{Objectives}

The current economic and social model needs to be reoriented toward more sustainable models, where Social Economy firms can be more efficient and sustainable. It is fundamental to delve into the management of Social Economy entities and explain their behaviour and contribution to sustainability. Therefore, the aim of this study is to present a theoretical model that shows the functioning mechanisms of Social Economy entities and associates the principles that govern this type of companies with certain organisational values. Such values, in turn, are specified in certain human resource management practices that have proved successful in the search for economic, social and environmental sustainability.

As a starting proposition, we consider that Social Economy entities are a sustainable model, where their principles and values influence such sustainability. The setting of a social entity begins with the establishment of some principles that are regulated by the applicable legislation. These principles pursue a clear general economic and social interest and aim to combine social progress and business efficiency. However, it is their values which grant entities their distinctive personality, values with a clear orientation toward business ethics and social responsibility. Our proposition is based on the fact that the business model of Social Economy is founded on organisational principles and values that are in line with the concept of economic, social and environmental sustainability. These values guide Social Economy firms toward the attainment of the 2030 Agenda Goals, especially regarding full and quality employment for everyone, with the aim of generating sustained, inclusive and sustainable growth (Steiner, 2018).

\section{The methodological design}

The methodology used is based on the use of qualitative techniques, and it was designed in two phases.

The first phase consisted in narrowing down the target population of the study, resorting to the "Relevant Social Economy Firms 2016-2017" report, published at the beginning of 2018 by the Spanish Business Confederacy of Social Economy (CEPES, 2018), which is the highest representative entity of Social Economy in Spain. Such report gathers data about the turnover, employment, territory and activity of 848 Social Economy entities, classified by their legal form from highest to lowest turnover, thus providing a representative sample of the different entities that compose the Spanish Social Economy. The entities gathered in such report pose 
an important narrowing down of the sector and, thus, a great opportunity to determine which organisational values are the most significant for these firms.

In the second phase, an analysis was performed on the corporate values of Social Economy firms from the sample provided by CEPES. The organisational values of each of the organisations were checked through their corporate website, obtaining data about the organisational values of 262 Social Economy entities. To this end, we used the NVivo 11 software for Windows, which was designed to organise, analyse and find perspectives of non-structured or qualitative data, such as interviews, answers to open questions, articles, content in social networks and, in our case, content in corporate websites. Among the different tools of NVivo 11, we used the word frequency check, through which the software allows enumerating the words that appear more frequently in resources, nodes, annotations and selected sets. To handle the obtained data, we previously defined the reference nodes, considering nodes as correlations of references about a topic, place, person or any other particular area of interest. The references were compiled by reading the resources, such as interviews and specific groups of interest, and subsequently classifying the information by categories in the relevant nodes; in our case, each organisational value was considered as a node and, therefore, defined for it to be recognised by the application in the dataset either by the word or by the definition.

\section{Results}

The obtained results showed the three organisational values that appear most frequently in the different Social Economy entities analysed: perceived support, respect and responsibility.

Perceived support is based on the theory of social exchange (Mael \& Ashforth, 1992), according to which the workers tend to change effort and dedication for their organisation through tangible incentives, such as competitive salaries, social benefits or emotional incentives (e.g., appreciation or recognition). Therefore, perceived organisational support has a direct influence on the business relationship between the company and the employee, generating an important bond (Rocha, 2015).

Respect is present within organisations and improves their effective communication, as well as the management of diversity, which is understood as the heterogeneity in the distribution of the personal characteristics of the members of an organisation without disregarding their cultural differences. Thus, workers are valued by their capacities, competencies, skills and knowledge, excluding from this valuation aspects such as gender, age and ethnicity, among others (Järlström et al., 2018).

Responsibility governs the creation of a business model that prioritises the concept of sustainability, adding value and placing economic, social and environmental aspects at the same level. Organisations that regard this organisational value as important assume that their economic profit is not above the working conditions of their employees (Wagner, 2013).

The obtained results are coherent with the existing literature. Authors such as Connelly et al. (2011) explored the potential of the values promoted by Social Economy in the search for a more sustainable environment, through business initiatives aimed at creating a synthesis of economic, social and environmental goals that lead us to achieve a structural change in 
the way in which goods and services are produced, accessed and consumed in our society. Therefore, as the main contribution of this study, we propose that the organisational values of Perceived Support, Respect and Responsibility are directly associated with Social Economy entities and their economic, social and environmental sustainability.

\section{Original Value and Contribution}

The present study contributes to the existing literature from a double perspective. Firstly, it proposes a theoretical model that aims to show the management mechanisms that can lead Social Economy entities to attain better results in corporate sustainability: lower business destruction rate and lower employment destruction rate during the crisis, greater inclusion and social cohesion, better results in environmental sustainability, etc. Such model proposed that the principles on which Social Economy is founded are transformed into corporate values that imbue their strategy and way of acting. Among these values, most of the analysed entities show Perceived Support, Respect and Responsibility. Each of these values are, in turn, instrumentalised in the business management practice in a very diverse manner, although our model is only focused on how they are converted to a certain way of managing people in Social Economy entities. It is the Human Resource Management practices, imbued with the organisational values, the ones that allow for better results in sustainability.

Secondly, our study analysed a sample of Social Economy entities, through which we determined the three most representative values in Social Economy. It is important to highlight the relevance of the human resource in the development of each of these three values in this type of entities, which place their workers at the core of their management, granting them greater participation, both in the management and in decision making. This guarantees stable and quality employment, ensuring the necessary stability that enhances the commitment between employee and employer. Likewise, the entities analysed in our study are important employment enclaves for population sectors with serious difficulties to access a quality job. The management of the heterogeneity of an organisation's workforce is fundamental in Social Economy entities, where aspects such as equality and reconciliation of the work and personal life become a priority in their human resource management.

Keywords: Social Economy, Organisational values, Sustainability, Human Resource Management. 


\section{Introducción}

Tras la crisis económica y financiera de 2008, cada vez más empresas parecen estar dispuestas a considerar que el modelo económico y social conocido hasta el momento está abocado al fracaso. La maximización de beneficios como único objetivo empresarial ha sido cuestionada tanto desde el ámbito académico como desde las propias empresas. Por ejemplo, recientemente, la Business Roundtable (que agrupa a 181 grandes organizaciones de Estados Unidos como ExxonMobil, JPMorgan Chase, Apple o Walmart, entre otras) ha declarado que el objetivo de las empresas no es sólo la maximización del beneficio, sino establecer el compromiso para crear valor para todos sus stakeholders (Business Roundtable An Association, 2019). En este debate económico, político y social, comienza a considerarse que los actuales recursos naturales, sociales y financieros son insuficientes para satisfacer a la población, así como el intenso estilo de vida de los países industrializados, que afecta considerablemente al agotamiento de dichos recursos (Ehnert, 2009).

El actual modelo económico y social necesita una reorientación hacia modelos más sostenibles, donde las empresas de Economía Social pueden mostrarse más eficaces. Por ejemplo, durante la crisis económica de 2008 en Europa, el número de empresas de Economía Social que se destruyeron fue sensiblemente inferior al del resto de empresas. Del mismo modo, las empresas de Economía Social destruyeron menos empleo que las empresas del sector capitalista en el periodo de crisis, basado fundamentalmente en la transformación de empresas mercantiles en empresas de Economía Social, evitando así su cierre y salvando a través de esta transformación o reconversión miles de puestos de trabajo en Europa (COCETA, 2018).

La necesidad de profundizar en la gestión de las entidades de Economía Social para explicar su comportamiento sostenible obliga a desarrollar un modelo teórico que explique de qué manera contribuyen estas organizaciones a la sostenibilidad. Por tanto, el objetivo de esta investigación es presentar un modelo teórico que exponga los mecanismos de funcionamiento de las entidades de Economía Social. Este modelo teórico trata de vincular los principios que fundamentan este tipo de entidades con determinados valores organizativos que están incorporados en su cultura organizativa. Estos valores, a su vez, se concretan en determinadas prácticas de recursos humanos que se han mostrado exitosas en la búsqueda de una sostenibilidad económica, social y medioambiental.

Consideraremos por tanto, como proposición de partida que las entidades de Economía Social son un modelo sostenible, donde sus principios y valores van a incidir en dicha sostenibilidad (Smith et al., 2013). La configuración de una entidad social tiene como punto de inicio la redacción de unos principios que vienen regulados por la normativa aplicable, estos principios persiguen un claro interés general tanto económico como social e intentan aunar progreso social y eficiencia empresarial. Pero son sus valores los que dotan a la entidad de una personalidad distintiva, valores con una clara orientación hacia la ética empresarial y a la responsabilidad social. Nuestra propuesta se basa en el hecho de que el modelo de empresa de Economía Social está asentado en principios y valores organizativos que están alineados con el concepto de sostenibilidad económica, social y ambiental (Sunley \& Pinch, 2012). Estos 
valores orientan a las empresas de Economía Social hacia la consecución de los objetivos de la Agenda 2030, especialmente cuando se trata de alcanzar el pleno empleo o asegurar el trabajo decente para todas las personas con el fin de generar un crecimiento sostenido, inclusivo y sostenible (Steiner, 2018).

\section{Marco Teórico}

La Economía Social intenta maximizar el bienestar social en general a través de un análisis económico basado en las personas, primando por tanto el bienestar social sobre el económico. Borgaza et al. (2012) consideran a la Economía Social como un sistema económico avanzado centrado en el desarrollo social que juega un papel crucial en el reparto de la riqueza y la reducción de las desigualdades sociales.

En la Unión Europea existen actualmente más de 2,8 millones empresas de Economía Social, que en 2015 empleaban a 13,6 millones de trabajadores y representan el 8\% del PIB comunitario. La Economía Social Europea ha ido ganando en relevancia con el paso de los años. Ello se evidencia en que desde el año 2000 las instituciones europeas han desarrollado más de 200 documentos oficiales, abordando temas tan importantes como la creación de empleo sostenible, los servicios sociales, la innovación social, la cohesión social, el emprendimiento, entre otros.

\subsection{Principios y Valores de la Economía Social}

El actual panorama sitúa a la sostenibilidad empresarial en una posición clave del actual modelo de negocio, abriéndose a nuevas oportunidades y planteando nuevos retos, tanto para las organizaciones como para la sociedad en su conjunto (Greening \& Turban, 2000). En este contexto, donde la actual sociedad se enfrenta a desafíos tales como el cambio climático, la erradicación de la pobreza y la constante búsqueda del equilibrio entre progreso económico y social, las empresas comprometidas con la sostenibilidad están obteniendo excelentes resultados empresariales, debido a su adecuada gestión de los recursos y relación con los stakeholders (Mariappanadar, 2014). Partiendo de que la sostenibilidad es un proceso y no un estado (Jackson, 2012), se hace necesario conceptualizar y delimitar dicho término. La sostenibilidad es considerada como el equilibrio entre el consumo y la regeneración de recursos, donde las organizaciones se comprometen a regenerar y desarrollar los recursos que ellos mismos consumen hoy y necesitarán en el futuro (Ehnert, 2009). El concepto de sostenibilidad permite establecer las bases para superar con éxito los actuales desafíos de la sociedad, ubicando en el centro de la cultura empresarial los términos de sostenibilidad individual, organizacional y social, niveles íntimamente relacionados (Docherty et al., 2002). En este sentido la búsqueda de la sostenibilidad aportará a las organizaciones la maximización de su capital económico, social y ambiental (Elkington, 1994), determinando tres actores fundamentales para conseguir la 
sostenibilidad de las empresas: los beneficios económicos (Profits), las personas (People) y el planeta (Planet). Estos tres actores son conocidos como las tres Ps de la sostenibilidad.

Las entidades de Economía Social cuentan con un conjunto de principios y valores organizativos desde su constitución que promueven y facilitan la sostenibilidad económica, social y medioambiental. Entendemos que los principios de este tipo de entidades son el conjunto de parámetros que orientan su funcionamiento y que es a través de sus valores donde se establecen el conjunto de normas o cualidades que rigen el comportamiento de su capital humano, estableciendo las variables que influyen en su comportamiento y su capacidad de establecer las estrategias que fortalecen la identidad de las entidades de Economía Social (Chaves \& Sajardo-Moreno, 2004).

Existe cierto soporte teórico para argumentar que las entidades de Economía Social están más orientadas a gestionar sus recursos de forma sostenible (Ehrgott et al., 2011), ya que proporcionan soluciones innovadoras a los desafíos económicos, sociales y medioambientales, además de introducir en la economía capitalista valores como la solidaridad, equidad y gobernanza democrática (Dacheux \& Goujon, 2011). La Social Economy Europe publicó en 2015 el Libro Blanco de la Economía Social, en el que se recogen los principios rectores de las entidades de Economía Social que operan en el marco de la Unión Europa. Estos principios marcan la cultura y los valores de las entidades de Economía Social, ofreciendo otra forma de producir, emprender, gestionar y consumir, en definitiva, otra forma de entender la economía y la empresa. Estos principios son los siguientes: (1)primacía de la persona y del objeto social sobre el capital, (2)adhesión voluntaria y abierta, (3)control democrático por sus miembros, (4)conjunción de los intereses de los miembros usuarios y del interés general, (5)defensa y aplicación de los principios de solidaridad y responsabilidad, (6)autonomía de gestión e independencia respecto a los poderes públicos y (7)destino de la mayoría de los excedentes a la consecución de objetivos a favor del desarrollo sostenible, del interés de los servicios a los mismos y del interés general.

Estos principios generales se concretan o especifican en determinados valores organizativos que les otorgan un valor añadido, como puede ser la creación de puestos de trabajo de mayor estabilidad, calidad y flexibilidad interna para el mantenimiento de los mismos, la solidaridad y la vinculación con el entorno, fomentando el desarrollo local y la no deslocalización y finalmente la inclusión sociolaboral de personas con, diversidad funcional, pertenecientes a minorías étnicas y/o en riesgo de exclusión social (Karnani, 2011). La última crisis financiera y económica de 2008, afectó no sólo a empresas y trabajadores, sino también al modelo económico general basado en el crecimiento continuado e incontrolado. En este escenario las empresas de Economía Social proponen unos principios que consiguen paliar las deficiencias que han azotado al sector económico y empresarial.

En el contexto organizacional, autores como George \& Jones (1997) consideran los valores de una organización como normas que definen sus objetivos y la forma de alcanzarlos. Ambos autores sostienen además que los valores de una organización, su misión y visión determinan y condicionan el comportamiento de los individuos que la forman. Para Kaptein \& Wempe (2002), los valores fundamentales desarrollan la misión y la visión, describiendo patrones específicos para su logro y son considerados estándares de comportamiento empresarial para los individuos de una organización. 
Estos valores corporativos pueden referirse a la eficacia y eficiencia de la organización 0 pueden incluir explícitamente valores éticos. Melé (2005) considera que cuando los valores de una organización están alineados con los de sus empleados, fomentan la aparición de comportamientos morales que benefician tanto a empleado como a empresa. Entre los valores organizativos más presentes en las entidades de Economía Social destacan la solidaridad, la democracia, la igualdad, el respeto, el compromiso y la sostenibilidad, los cuales reflejan los principios de primacía de la persona, promoción del interés general, equilibrio económico y social y finalmente la gestión democrática (Liger et al., 2016). Autores como Defourny \& Nyssens (2012) sostienen que los valores que contienen este tipo de entidades marcan sus objetivos de marcado carácter social, es decir, la actividad empresarial es un medio para desarrollar una labor social que va más allá del puro mercantilismo. Estos valores organizativos suponen el ADN de las entidades de Economía Social. La identificación de estas entidades con ciertos valores las diferencia de las empresas del sector tradicional. En concreto la identificación de estos valores condiciona y determina la posición de la entidad ante el reto de la sostenibilidad, en sus tres dimensiones económica, social y ambiental. No obstante, estos valores organizativos no surgen espontáneamente, deben inspirar la misión y visión, diseño de estructura y modo de organización de las empresas de Economía Social (Melé, 2012), concretamente a través de sus prácticas de Gestión de Recursos Humanos, que pasamos a explorar a continuación.

\subsection{Prácticas de Gestión de Recursos Humanos}

De entre las diferentes funciones de la empresa, la Gestión de Recursos Humanos (GRRHH) es de las que mayor interés está despertando. Ello se debe principalmente a que académicos y profesionales han identificado el capital humano como un recurso estratégico de las organizaciones, que les permite desarrollar capacidades que contribuyen a su rendimiento organizativo (Huselid, 1995; Pfeffer, 2005). En los últimos años ha habido un creciente interés en el estudio de la GRRHH, dando lugar a la llamada gestión humana socialmente responsable. Esta gestión responsable de los recursos humanos se ha convertido en una prioridad estratégica para muchas empresas que ven como aumenta, de esta forma, el nivel de compromiso, motivación y desempeño de sus empleados (Barrena-Martínez et al., 2017).

Concretamente, la Economía Social está caracterizada por una gestión humana socialmente responsable, orientada a desarrollar el bienestar y la solidaridad a través de la información, la formación, la participación, las condiciones de trabajo, la salud y la seguridad laboral, la igualdad de oportunidades y la conciliación de la vida laboral y familiar (Bustamante Salazar, 2019). De entre todas las prácticas de GRRHH disponibles, nuestra investigación se centrará en tres prácticas concretas, la selección de personal, la formación y desarrollo profesional y, finalmente, la gestión de la diversidad, debido fundamentalmente a que son las que mejor muestran la vinculación entre los valores organizativos de las entidades de Economía Social y el impacto en la sostenibilidad de la organización (Florea et al., 2013). Consideramos que las organizaciones de Economía Social que están llevando a cabo esta gestión humana socialmen- 
te responsable desarrollan prácticas de gestión que aumentan el rendimiento de sus empleados (Cardoso et al., 2012).

Las prácticas de afectación buscan la incorporación del individuo a las organizaciones y están integradas por los procesos de reclutamiento, selección y orientación de sus trabajadores (Breaugh, 2008). Estas prácticas habitualmente son abordadas como un conjunto pues están fuertemente interrelacionadas. El reclutamiento es definido como la búsqueda de los candidatos potenciales en cantidad y calidad suficiente que permita posteriormente a la organización seleccionar al candidato más apropiado. En el proceso de selección se recopila la información de los candidatos para posteriormente evaluarlos y decidir a quién contratar. Estos procesos no pueden tratarse de forma separada y vienen complementados por la orientación del candidato contratado, a través de la cual se introducen a los nuevos empleados en la organización, ubicándolo en su lugar de trabajo y comunicándole las relaciones e interdependencias con otros puestos y compañeros. El proceso de reclutamiento y selección de las organizaciones está en constante cambio y evolución, debido a la continua evolución de la sociedad y la empresa. Los perfiles que las empresas requieren van evolucionando así como las preferencias y necesidades de los empleados (Breaugh, 2008).

Las prácticas de desarrollo de una organización son el proceso a través del cual sus empleados progresan a través de una serie de etapas caracterizadas por distintas tareas de desarrollo, actividades y relaciones (Mayo \& Gispert Ramis, 2000). Las prácticas de desarrollo están formadas por la formación y el diseño de la carrera profesional de los empleados. En concreto la formación tiene entre sus objetivos el mantenimiento de las competencias de la plantilla, la cual tiende a sufrir un proceso de obsolescencia de sus conocimientos, habilidades y destrezas. Para evitar dicha obsolescencia, las organizaciones diseñan planes de formación que pueden fomentar la polivalencia del recurso humano. Así mismo, entendemos que la carrera profesional de un empleado como la sucesión de actividades profesionales desempeñados por una persona a lo largo de su vida en una o diferentes organizaciones (Greenhaus et al., 2010). Conner \& Prahalad (1996) destacaron el impacto que ejerce la formación y la carrera profesional de los trabajadores sobre el desarrollo de la actividad productiva de la organización. La organización debe contar con empleados formados acorde con las exigencias de los puestos de trabajo. Por ello la formación debe tener cierta orientación proactiva, de forma que se ofrezca al empleado la posibilidad de formarse y adquirir nuevos conocimientos y habilidades que pondrá en práctica en un futuro o bien que podrán ser empleados en otro puesto de trabajo en caso de que la organización requiera cierta flexibilidad funcional. A través de la formación la organización busca aumentar la flexibilidad funcional de su capital humano, con la ventaja de poder ubicarlo en otros puestos de trabajo cuando sea requerido (Basterretxea \& Albizu, 2010).

La tercera práctica que abordamos es la gestión de la diversidad, que permite a las organizaciones nutrirse de las diferencias individuales para que sumen de forma conjunta a proyectos comunes. Hay que integrar para crecer, enfatizando en la variabilidad individual, valorando el aporte individual sin importar las características personales del individuo (Pless \& Maak, 2004). Para ello es necesario un compromiso real de la dirección de la empresa y las perso- 
nas implicadas en la toma de decisiones, conscientes de que la diversidad además de integrar aspectos tan relevantes como la cultura, la religión, el género y las diferencias funcionales, también puede generar mayores niveles de rendimiento e innovación. El término diversidad puede ser considerado como una propiedad de todo grupo donde prima su heterogeneidad en función de las características personales de los miembros que lo componen (Martín Alcázar et al., 2013). Podemos considerar la gestión de la diversidad como una importante palanca de transformación que distingue a las organizaciones más respetuosas, convirtiéndolas además en una entidad más innovadora, competitiva y con un mayor atractivo empleador. La organización debe generar condiciones que faciliten el empoderamiento de los empleados que la forman, así como planificar adecuadamente atendiendo a sus características personales, integrando el respeto a la característica individual en la política y cultura de la empresa, para finalmente eliminar cualquier proceso, comportamiento o acción que suponga una discriminación directa o indirecta hacia su capital humano.

\section{Metodología}

La globalización ha supuesto un serio desafío para la lograr y mantener la sostenibilidad de las organizaciones, debido fundamentalmente a la heterogeneidad de sus recursos humanos (Stone et al., 2007). Schramm (2011) sostiene que la sostenibilidad de una organización está estrechamente relacionada con la forma que la que sus empleados trabajan, y más concretamente cómo los valores individuales afectan al desarrollo de la tarea. Sin embargo, somos conscientes del vacío existente sobre qué valores concretos apoyan e inciden en la sostenibilidad de las organizaciones.

A principios de 2018 la Confederación Empresarial Española de Economía Social (CEPES, 2018), máxima entidad representativa de la Economía Social española, publica por undécimo año consecutivo, el informe "Empresas relevantes de la Economía Social 2016-2017", a través del cual se recogen los datos relativos a la facturación, empleo, ámbito territorial, actividad de 848 entidades de Economía Social, clasificadas por su forma jurídica y ordenadas de mayor a menor facturación económica. Este informe nos ofrece una muestra representativa de las diferentes entidades que conforman la Economía Social española. Las entidades recogidas en dicho informe suponen una importante acotación al sector y por tanto una gran oportunidad para determinar qué valores organizacionales son los más significativos para estas entidades seleccionadas. Hemos realizado un estudio sobre los valores corporativos de las entidades de Economía Social a partir de la muestra ofrecida por CEPES. A partir de dicha muestra, se han consultado los datos de cada una de las organizaciones a través de su web corporativa.

De las 848 entidades que conforman el informe de CEPES hemos encontrado información referente a sus valores corporativos en la web de 262 entidades, lo que supone una tasa del $31 \%$ aproximadamente.

Los datos recabados de esas 262 organizaciones son de índole cualitativa por lo que los hemos tratado con el software NVivo 11 for Windows, diseñado para organizar, analizar y en- 
contrar perspectivas de datos no estructurados o cualitativos, tales como entrevistas, respuestas a encuestas con preguntas abiertas, artículos, contenido en redes sociales y en nuestro caso contenido en webs corporativas. De entre las diferentes herramientas que nos ofrece el software NVivo 11 hemos empleado la consulta de frecuencia de palabras, a través de la cual el software nos permite enumerar las palabras que se presentan con más frecuencia tanto en recursos, nodos, anotaciones y conjuntos seleccionados. Para trabajar los datos obtenidos, previamente hemos definido los nodos de referencia, considerando como nodo una colección de referencias acerca de un tema, lugar, persona o cualquier otra área de interés en especial. Las referencias se recopilan leyendo los recursos, como entrevistas y grupos de interés específicos, y posteriormente clasificando la información por categoría en los nodos relevantes, en nuestro caso cada valor organizativo ha sido considerado como nodo y por tanto definido para que la aplicación reconozca en el conjunto de datos bien la palabra o la propia definición.

\section{Resultados}

Como resultado final el software NVivo 11 for Windows nos ofrece una gráfica en la que aparecen el número de repeticiones de cada valor organizativo o definición del mismo contenida en cada nodo del conjunto de datos estudiados (Tabla 1).

\section{Tabla 1. Valores organizativos más representativos en la Economía Social}

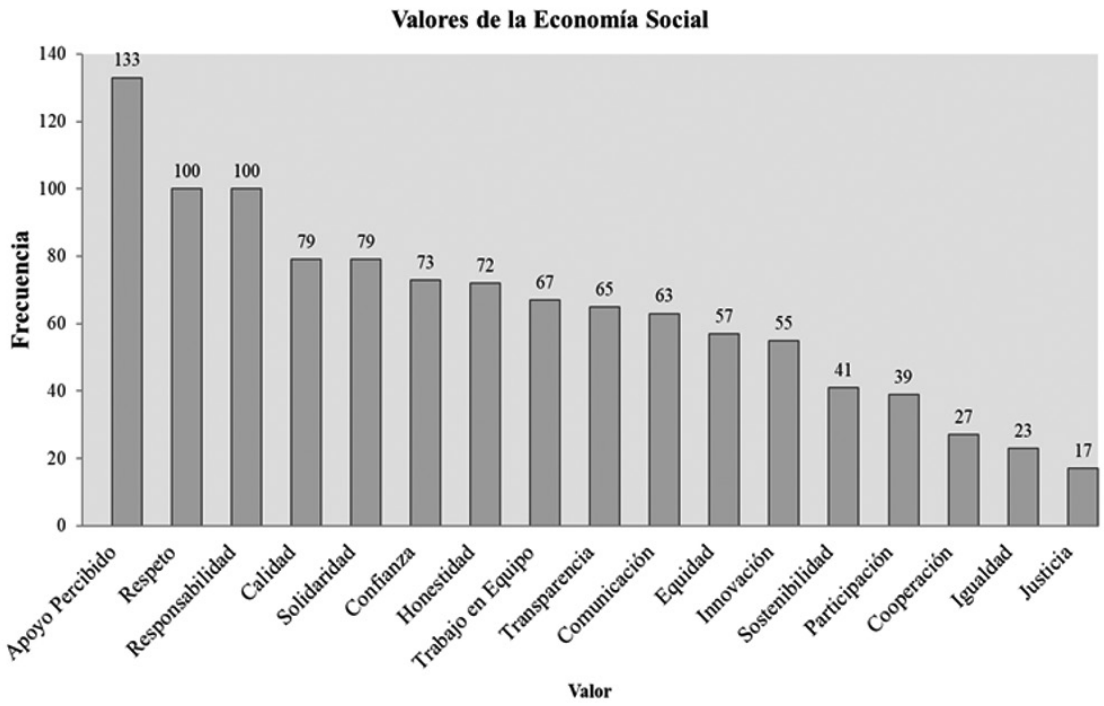

Fuente: Elaboración propia. 
Como se desprende del gráfico anterior el programa nos ofrece como resultado los 17 valores organizativos más repetidos en la muestra de 262 entidades estudiadas, siendo los diez valores más relevantes los consignados en la Tabla 2.

\section{Tabla 2. Valores más relevantes}

\begin{tabular}{|c|c|}
\hline Relevancia & Valor \\
\hline 10 & Apoyo Percibido \\
\hline $2^{0}$ & Respeto \\
\hline $3^{0}$ & Responsabilidad \\
\hline $4^{0}$ & Calidad \\
\hline 50 & Solidaridad \\
\hline 60 & Confianza \\
\hline $7^{0}$ & Honestidad \\
\hline $8^{\circ}$ & Trabajo en Equipo \\
\hline go & Transparencia \\
\hline 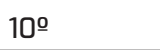 & Comunicación \\
\hline
\end{tabular}

Fuente: Elaboración propia.

Acotando los datos obtenidos podemos observar cómo son tres los valores más frecuentes en las entidades de Economía Social, que además están presentes en casi la mitad de la muestra estudiada. Los valores Apoyo Percibido, Respeto y Responsabilidad son los más citados, con más de 130 referencias en el primer caso y 100 en los dos siguientes.

Una vez determinados los tres valores organizativos que más se repiten en las diferentes entidades estudiadas, se hace necesaria la conceptualización de cada uno de ellos:

El valor con mayor presencia en el conjunto de entidades estudiadas es el valor Apoyo Percibido. El concepto de apoyo percibido proviene de la teoría del apoyo organizacional (Eisenberger et al., 1986) y se refiere a la valoración que hace un empleado sobre cómo la organización valora sus contribuciones y se preocupa por su bienestar. De esta forma los empleados de una organización consideran que la empresa valora sus esfuerzos y aportaciones, aumentando por tanto el compromiso del empleado con la organización, lo que incide positivamente en el rendimiento del empleado ante situaciones críticas o estratégicas de la organización, tales como una reorientación estratégica o una reestructuración de plantilla (Lee \& Peccei, 2007). El apoyo percibido está basado en la teoría del intercambio social (Mael \& Ashforth, 1992), en la que los trabajadores tienden a cambiar esfuerzo y dedicación con su organización a través de incentivos tangibles, como pueden ser salarios competitivos, beneficios sociales o incentivos emocionales como la aprobación o el reconocimiento. Así pues, el apoyo organizacional 
percibido influye directamente en la relación laboral entre empresa y empleado generando un importante vínculo (Rocha, 2015).

El segundo valor que nos encontramos es el valor Respeto, el cual incluye la consideración y deferencia. A nivel organizacional el respeto busca la convivencia armónica en el trabajo, que incide en el rendimiento del empleado y la organización (Rogers \& Ashforth, 2017). Atendiendo al modelo "ROC" (Respect, Openness, Continuity) propuesto por De Prins et al., (2014), el respeto en las organizaciones también mejora la comunicación laboral así como el trabajo en equipo, siendo un importante generador de confianza y compromiso entre empresa y empleado. Consideramos que la relación laboral que se produce en una organización basada en el respeto y la comprensión generará mejores relaciones a largo plazo entre empleador y empleado (Huiskamp \& Kluytmans, 2004). Asimismo, el respeto dentro de las organizaciones mejorará la comunicación efectiva de la misma, la gestión de la diversidad, entendida ésta como la heterogeneidad en la distribución de las características personales de los miembros de una organización sin dejar atrás sus diferencias culturales. Valorando a las personas por sus capacidades, competencias, habilidades y conocimientos, y dejando fuera de tal valoración cuestiones como el género, la edad, la etnia, entre otras (Järlström et al., 2018).

El tercer valor que nos ofrece la muestra estudiada es el valor Responsabilidad. Por responsabilidad se entiende la capacidad existente para reconocer y aceptar las consecuencias de un hecho realizado libremente de forma individual o en grupo (Gill, 2012). La actual sociedad demanda que las personas y entidades actúen de forma responsable, atendiendo a criterios éticos y morales (Ketola, 2008). Este valor rige la creación de un modelo de empresa que prioriza el concepto de sostenibilidad, dando valor y colocando al mismo nivel aspectos económicos, sociales y ambientales. Las organizaciones que dan importancia a este valor organizativo, la responsabilidad, asumen que su beneficio económico no está por encima de las condiciones laborales de sus empleados (Wagner, 2013). Diseñando por tanto políticas encaminadas a mejorar las condiciones de trabajo, la calidad de vida de sus empleados a través de la seguridad en el empleo, generando procesos participativos y de comunicación abierta, todo ello encaminado a mejorar la relación laboral y consecuentemente la productividad y la competitividad de la empresa. The World Economic Forum (2018) ha destacado como tras la crisis económica de 2008 la principal preocupación de los trabajadores de nuestra sociedad es encontrar un empleo y mantenerlo. Añadiendo además que el deterioro del mercado laboral ha provocado la aparición de factores como la insatisfacción laboral, aumento del absentismo y del microabsentismo de la mano de obra, que trae importantes consecuencias para las organizaciones y es por ello que estas deben asumir su responsabilidad hacía los empleados y favorecer una relación laboral con un alto componente de seguridad en el empleo.

\section{Discusión}

Como consecuencia de todo lo anteriormente expuesto, la presente investigación presenta como proposición de partida que las entidades de Economía Social cuentan con un conjunto de 
valores organizativos que desde su constitución favorecen y facilitan la sostenibilidad económica, social y medioambiental. Estudios como el elaborado por Connelly et al. (2011) exploran el potencial de los valores que promueven la Economía Social en la búsqueda de un entorno más sostenible, a través de iniciativas empresariales que se esfuerzan por crear una síntesis de objetivos económicos, sociales y ambientales que logren un cambio estructural en la forma en la que se producen, acceden y consumen los bienes y servicios de nuestra sociedad. Por tanto, como primera proposición, planteamos la relación directa existente entre los valores organizativos Apoyo Percibido, Respeto y Responsabilidad de las entidades de Economía Social y su sostenibilidad económica, social y ambiental.

\section{Figura 1. Proposición principal de investigación}

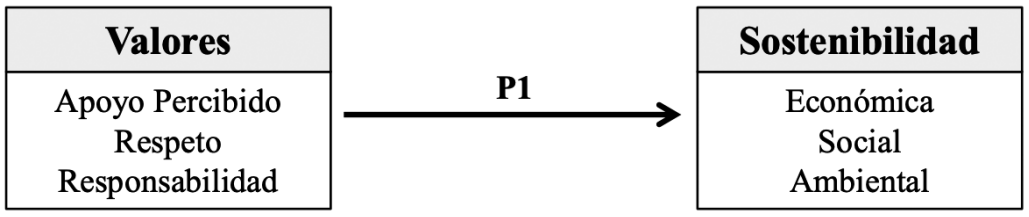

\section{Entidades de Economía Social}

Fuente: Elaboración propia

PROPOSICIÓN 1: Los valores organizativos: Apoyo Percibido, Respeto y Responsabilidad de las entidades de Economía Social determinan el compromiso de dichas entidades con su sostenibilidad.

Un segundo planteamiento de la presente investigación aborda la posible incidencia de los valores organizativos de las entidades de Economía Social sobre el diseño de sus prácticas de recursos humanos, contemplando la GRRHH como variable mediadora de la relación existente entre dichos valores y la sostenibilidad.

En las entidades de Economía Social la formulación de cada valor se hace con la intención de conseguir un objetivo para la propia organización y personas que la componen (Napathorn, 2018). De forma que los valores definen unos objetivos concretos, los cuales se verían favorecidos por diferentes prácticas de GRRHH. Tal y como recogen Schuler \& Jackson (2014) las empresas están usando la gestión de sus recursos humanos para mejorar su organización estratégica y no sólo en base a la rentabilidad económica. La gestión eficiente de la plantilla en una entidad de Economía Social es esencial, debido a varios motivos: en primer lugar, la complejidad de las figuras laborales que la conforman, ya que en ellas conviven la figura del socio-trabajador con la del trabajador en el caso de las cooperativas y sociedades laborales. 0 como en el caso de las empresas de inserción o centros especiales de empleo, donde entra en juego la figura de los empleados de estructura o equipo técnico. Y en segundo lugar, el grado de 
responsabilidad que asume la entidad social sobre sus empleados, quienes ocupan un puesto central en el conjunto de políticas que llevan a cabo estas entidades (Guillén \& Cespedes-Lorente, 2003).

Por todo ello planteamos tres nuevas proposiciones vinculando los valores organizativos más frecuentes en las entidades de Economía Social estudiadas con determinadas prácticas de GRRHH. Abordamos cómo influye cada valor en el diseño de la práctica de recursos humanos o cómo estas entidades usan su la gestión de su capital humano para reforzar su gestión en valores, es decir, las prácticas de GRRHH son el instrumento que usan las empresas de Economía Social para generar un modelo empresarial distinto, cohesionado y sostenible.

\section{Figura 2. Valores y Prácticas de Gestión de Recursos Humanos}

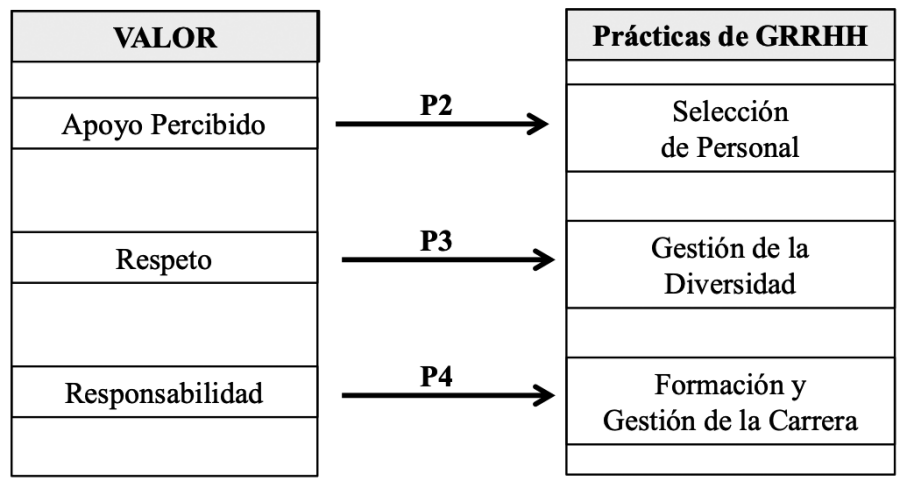

Entidades de Economía Social

Fuente: Elaboración propia.

En primer lugar, establecemos una relación directa entre el valor organizativo de Apoyo Percibido con las Prácticas de Selección de personal. A nivel organizativo consideramos que el compromiso que establece una organización con sus empleados genera una imagen de la empresa que la hace más atractiva, fomentando considerablemente su imagen de empleador y aumentando su capacidad de atraer talento (App et al., 2012). El proceso de selección supone la toma de decisión por parte de la organización de a qué personas contratar e introducir en su estructura, basada en una serie de procedimientos y técnicas encaminadas a asegurar el éxito del ajuste del candidato al puesto de trabajo y a la propia organización (Tabiu \& Nura, 2013). Desde las entidades de Economía Social se hace especial hincapié en establecer un procedimiento sobre el proceso de selección. Consideramos que el compromiso que se establece en una organización social es más un contrato psicológico que una mera forma contractual, es decir estas entidades buscan la vinculación con el individuo ofreciéndole apoyo para conseguir la alineación de intereses entre empresa social y empleado a través de un contrato psicológico, 
que vas más allá de la obligación formal de desarrollar un trabajo: implica la existencia de un conjunto de expectativas recíprocas en la relación establecida entre la organización y el trabajador (App et al., 2012).

Es en este proceso de incorporación de los empleados a las entidades de Economía Social donde se asientan los cimientos del compromiso que se establece entre trabajador y su organización. Basado fundamentalmente en dos consideraciones, en primer lugar, entendemos que en el proceso de selección se tienen en cuenta valores intrínsecos de los candidatos, intentando identificar una alineación de dichos valores con los de la propia organización. En segundo lugar, estas entidades ofrecen desde el inicio una clara imagen organizativa, facilitando al futuro empleado su posición en la misma así como sus posibilidades de desarrollo, enfatizando en la centralidad del empleado en la gestión y en la toma de decisiones. Estas entidades no sólo reclutan y seleccionan en base a competencias, comprenden que la conducta individual del empleado está sujeta a normas morales de comportamiento, las cuales deben ser gestionadas por las entidades (Sharma \& Bhatnagar, 2016). Finalmente, consideramos que las prácticas de selección de personal en las entidades de Economía Social son un instrumento para proyectar su imagen al exterior, ya que emprende acciones para publicar su imagen, con el objetivo de atraer a candidatos altamente cualificados, seleccionarlos para su organización y ubicarlos en la misma (Bhattacharya et al., 2005). La gestión de recursos humanos sostenible puede ayudar a crear una marca de empleado atractiva o "employer branding" generadora de una ventaja competitiva sostenible (App et al., 2012), atrayendo y reteniendo a empleados con alto potencial que ven en la gestión humana de la entidad social un factor de atracción, que genera un compromiso mutuo entre empleador y empleado. A partir de lo expuesto podemos formular la siguiente proposición:

PROPOSICIÓN 2: El valor Apoyo Percibido mejora el proceso de selección de las entidades de Economía Social: favoreciendo la marca de empleador de la entidad y atrayendo a candidatos con un alto potencial.

Planteamos una tercera proposición basada en la relación directa entre el valor Respeto que muestra la organización hacía la diversidad y heterogeneidad de su fuerza de trabajo, que consideramos tiene una estrecha vinculación con la Gestión de la Diversidad dentro de las organizaciones sociales. La actual situación económica, financiera y tecnológica está fuertemente condicionada por el proceso de globalización que está sacudiendo a nuestra sociedad. Globalización que ha traído consigo importantes transformaciones en los procesos organizativos de las empresas, así como en los mercados donde éstas operan y que además han modificado la forma de trabajar y de estructurar nuestra vida social y familiar (Perkins, 2003). Es por tanto que las organizaciones actuales no pueden seguir funcionando con los mismos principios y valores que hace años: la sociedad ha cambiado haciéndose más diversa y heterogénea. Es por ello que las organizaciones deben adaptarse y saber integrar los cambios sociales en su estructura. Las organizaciones deben reconocer y gestionar la diversidad de su recurso humano, integrándola además en la formulación de su misión, visión y valores (DiSte- 
fano \& Maznevski, 2000). En esta línea, el pensamiento social considera necesaria la aparición de una economía inclusiva, donde las empresas asuman sus responsabilidades para mitigar: el cambio climático, las diferencias económico-sociales, así como mejorar la inclusión social y el bienestar, abandonando el individualismo para pasar a una forma de organización más comunitaria, abierta y colaborativa (Sengupta et al., 2018).

Algunas entidades de Economía Social se caracterizan por dar cabida a sectores de la población que tienen escasas oportunidades laborales, o bien tienen más complicado el acceso al mercado laboral de nuestra sociedad. En este sentido cabe destacar importantes grupos dentro del sector social, como son los Centros Especiales de Empleo que ocupan a personas con diversidad funcional, así como las Empresas de Inserción, destinadas básicamente a emplear a personas en riesgo de exclusión social y sectores de la población con escasos recursos. Por su parte las cooperativas de trabajo asociado, han supuesto un importante salvavidas para el empleo durante la última crisis económica. Empleados que vieron cómo la empresa en la que habían trabajado durante años decretaba el cierre se constituyeron como alternativa en cooperativa de trabajo, asumiendo la actividad productiva de la anterior empresa, pero con una forma de gestión y de gobierno más colaborativa (Estapé \& Torreguitart, 2014). Las entidades de Economía Social muestran un claro respeto hacia la igualdad de oportunidades dentro de sus organizaciones, igualdad no sólo de género entre hombre y mujeres, igualdad de oportunidades en busca de una cohesión social, donde el empleado es respetado, no sólo en sí mismo sino en su interacción con la sociedad, a través de un empleo digno, estable y de calidad, fomentando además una conciliación real de la vida personal, familiar y laboral.

Este valor tiene una alta presencia en la idiosincrasia de las entidades estudiadas, siendo fundamental para armonizar los intereses de individuo, empresa y sociedad, así como la interacción social y medioambiental. Consideramos que las entidades de Economía Social entienden el valor respeto como el reconocimiento de las particularidades de las personas que forman parte de su organización, y que aportan a su estructura una heterogeneidad que afectará a su forma de gestión y por ende a sus resultados, no sólo económico sino también social. En este sentido podemos afirmar que estas entidades se postulan como las organizaciones que más impacto tienen en la integración y cohesión social de nuestra sociedad (Longinos et al., 2012). De esta forma consideramos que las entidades de Economía Social muestran un claro respeto hacia los intereses de sus miembros, colando al mismo nivel aspectos económicos y sociales, por lo que plantemos la siguiente proposición:

PROPOSICIÓN 3: El valor Respeto favorece la gestión de la diversidad de las entidades de Economía Social: aumentando la heterogeneidad en su plantilla.

Finalmente, abordamos el tercer valor con mayor presencia en las entidades objeto de nuestra investigación, el valor Responsabilidad, a través del cual las organizaciones colocan a sus empleados en el centro de su gestión y otorgan especial importancia a las prácticas de GRRHH de Formación y Desarrollo Profesional en las entidades de Economía Social. El desarrollo profesional de una persona dentro de una organización se inicia con el proceso de formación, y se completa con el diseño de su carrera profesional, entendiendo a esta como la sucesión 
evolutiva de actividades laborales desempeñadas por una persona a lo largo de su vida en una misma o diferente organización. Hoy en día no podemos contemplar el término de carrera profesional sin considerar el impacto de las acciones formativas de la organización sobre sus empleados (Greenhaus et al., 2010).

El desarrollo profesional de un empleado en una organización pasa por fomentar sus conocimientos, habilidades y destrezas (Kamoche, 1996). La responsabilidad de una organización hacía sus empleados tiende a centrarse en el aprendizaje y la promoción de estos, con la finalidad de aumentar su sensación de seguridad. Ello a través de un mayor enfoque de responsabilidad social que potencia además la identificación corporativa del empleado hacía su empresa (Fenwick \& Bierema, 2008). En las entidades de Economía Social la formación pasa por el empoderamiento del empleado, considerando que un empleado formado es un empleado polivalente. Debido al principio de primacía de las personas sobre el capital, las empresas de Economía Social sitúan a sus empleados en el centro de su gestión, promoviendo su participación e integrándolos en los objetivos de la empresa, lo que inexorablemente modifica la gestión humana de la empresa, gestión basada fundamentalmente en valores éticos.

El valor responsabilidad se concreta ante los empleados a través de prácticas de GRRHH orientadas a promover su desarrollo personal y profesional, concretadas en acciones formativas que fomenten sus capacidades, conocimientos y habilidades, así como la transmisión de conocimientos entre empleados (Bamberger et al., 2014). Las organizaciones asumen la responsabilidad de conseguir que sus empleados estén seguros en sus puestos de trabajo. Esa responsabilidad les lleva a, fomentar el desarrollo personal y profesional de los mismos a través del diseño de planes de carrera donde la formación tiene un papel relevante. La diferencia fundamental con las prácticas de formación de las empresas que no son de Economía Social y por tanto no tienen incorporado el valor Responsabilidad en su ADN, es que las entidades de Economía Social se preocupan del desarrollo total del trabajador (socio o empleado), tanto en su faceta personal como profesional. Ya que las personas que forman parte de la entidad social son consideradas como fines en sí mismos y no medios o recursos para alcanzar los fines organizativos, los planes de formación están orientados al desarrollo profesional y también personal del trabajador, promoviendo su empowerment y elevando por tanto el nivel de vida de las personas y por ende fomentando la cohesión económica y social, así como el fomento de empleo estable y de calidad (Zhang et al., 2018). Entendemos que los planes de formación y desarrollo profesional de las organizaciones deben responder a las exigencias tanto del entorno como de los propios empleados, es por ello que las organizaciones asumen su responsabilidad hacía sus empleados, ofreciéndoles recursos para analizar y planificar estrategias de carrera a sus empleados, ayudándolos a explorar nuevas alternativas que consigan aumentar su formación, motivación y rendimiento (Mone, 2018).

El análisis anterior nos lleva a plantear la siguiente proposición:

PROPOSICIÓN 4: El valor Responsabilidad mejora el proceso de formación y desarrollo profesional de las entidades de Economía Social: la organización implica a sus empleados con la adquisición y desarrollo de nuevos conocimientos, habilidades y destrezas. 
La figura 3 recoge el modelo teórico completo que proponemos:

Figura 3. Modelo de investigación planteado

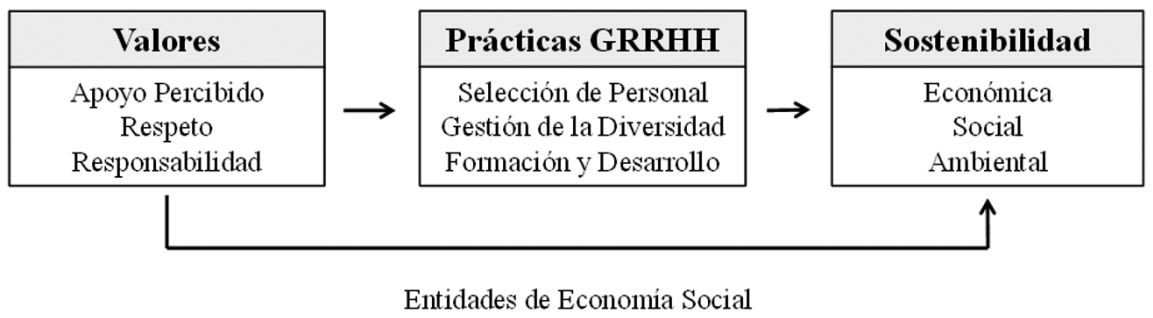

Fuente: Elaboración propia.

\section{Conclusiones}

Nuestro trabajo plantea un modelo teórico que trata de mostrar los mecanismos de gestión que pueden llevar a las entidades de Economía Social a obtener mejores resultados en sostenibilidad corporativa: menor tasa de destrucción de empresas y menor destrucción de empleo durante la crisis, mayor inclusión y cohesión social, mejores resultados en sostenibilidad ambiental, entre otros. Dicho modelo plantea que los principios en los que se fundamenta la Economía Social son convertidos en valores corporativos que impregnan su estrategia y forma de actuar. Entre esos valores destacan, en la mayoría de las entidades estudiadas, el valor Apoyo Percibido, el valor Respeto y el valor Responsabilidad. Cada uno de estos valores, a su vez, son instrumentalizados en la práctica de la gestión empresarial de forma muy diversa, pero nuestro modelo se centra en cómo se convierten en una determinada forma de gestionar las personas en las entidades de Economía Social. Son las prácticas de gestión de Recursos Humanos, imbuidas de los valores organizativos, las que hacen posible los mejores resultados en sostenibilidad.

El modelo social consigue aunar desarrollo económico y sostenibilidad, en sus tres vertientes económica, social y ambiental, generando modelos empresariales con una visión económica más social y solidaria, caracterizados por la búsqueda de la cohesión social y el respeto absoluto a los recursos naturales que emplea en el desarrollo de su actividad. Se fomenta además un estilo de vida y de desarrollo de la sociedad respetuoso con el ser humano y con el medio ambiente. Las entidades de Economía Social están constituidas al amparo de una serie de principios que favorecen su orientación hacía la sostenibilidad, a través del desarrollo de una serie de valores que las hacen diferentes, particulares y por ende sostenibles.

Nuestra investigación ha realizado el estudio de una muestra de entidades de Economía Social, a través del cual hemos determinado los tres valores organizativos más representativos en la Economía Social, tales como el Apoyo Percibido, Respeto y Responsabilidad. Es necesario 
destacar cómo en el desarrollo de cada uno de estos tres valores destaca la importancia que posee el recurso humano en este tipo de entidades, situando a sus empleados en el centro de su gestión, otorgándoles mayor participación, tanto en la gestión como en la toma de decisiones. Ofreciendo garantías de un empleo estable y de calidad, asegurando con ello una estabilidad necesaria que potencie el compromiso entre empleado y organización. Así mismo, vemos como las entidades objeto de estudio de nuestra investigación son importantes enclaves de empleo para sectores de la población con importantes dificultades para acceder a un empleo de calidad. La gestión de la heterogeneidad en una plantilla es fundamental en las entidades el sector de la Economía Social, donde aspectos como la igualdad y la conciliación de la vida laboral y familiar pasan a ser una prioridad en su GRRHH.

Finalmente, abordamos el impacto que tienen los tres valores organizativos, que hemos determinado en la muestra estudiada, sobre la GRRHH de las entidades de Economía Social, concretamente planteamos tres posibles relaciones entre cada valor y tres determinadas prácticas de Recursos Humanos, observando cómo cada valor ejerce una importante influencia en el diseño de esa determinada práctica. El modelo propuesto supone un avance en la comprensión del funcionamiento de las entidades de Economía Social y de los mecanismos que llevan a su éxito. Sustentado en cómo este tipo de entidades han combatido la crisis económica de una forma más sostenible que las empresas del mercado tradicional: manteniendo empleo, creando nuevas empresas y fomentando la cohesión social, que se ha visto deteriorada por el actual sistema económico y financiero, basado en la maximización del rendimiento como único índice de crecimiento (Jackson, 2016).

Nuestra investigación presenta una serie de limitaciones. La más importante es que la investigación sobre los valores corporativos se circunscribe a un único contexto geográfico, España, por lo que se hace necesario en futuras investigaciones aumentar la población de estudio. También cabe destacar como limitación que la información extraída para determinar los valores organizativos más representativos se ha llevado a cabo a través de las webs corporativas de las entidades. En este sentido debemos destacar que las empresas de Economía Social aún tienen un importante camino por recorrer a la hora de exponer sus productos, servicios e imagen en la red.

La presente investigación presenta como línea futura de investigación el planteamiento de una variable moderadora, como son las Relaciones de Empleo, formuladas por los autores Tsui \& Wu (2005). Consideramos que estas pueden incidir positiva y significativamente en la consecución de la sostenibilidad en las empresas de Economía Social. Nos planteamos si las relaciones de empleo condicionan el diseño e implantación de un determinado Sistema de Gestión de Recursos Humanos, es decir, nos cuestionamos si existen dentro de las entidades de Economía Social diversidad de prácticas de gestión de Recursos Humanos, las cuales a su vez tienen un especial impacto sobre la sostenibilidad de la entidad. Abordaremos el estudio de las relaciones de empleo en las entidades de Economía Social, entendiendo como relación de empleo el intercambio donde el empleador contrata fuerza de trabajo con la finalidad de producir bienes o servicios, para obtener un beneficio propio. Y donde el empleado presta sus servicios a cambio de una contraprestación, la cual puede o no ser solamente económica. 


\section{Bibliografía}

ALGORA JIMÉNEZ, J.M. (2011): “La Economía Social: Crisis y Recuperación Económica”, Historia Actual Online, 26, 133-140.

APP, S., MERK, J. \& BÜTTGEN, M. (2012): "Employer Branding: Sustainable HRM as a Competitive Advantage in the Market for High-Quality Employees", Management Revue, 23(3), 262278. Retrieved from http://www.jstor.org/stable/41783721.

BAMBERGER, P.A., BIRON, M. \& MESHOULAM, I. (2014): "Human resource strategy: Formulation, implementation, and impact, Second Edition", Human Resource Strategy: Formulation, Implementation, and Impact, Second Edition: Taylor and Francis. https://doi.org/10.4324/9780203075838.

BARRENA-MARTíNEZ, J., LÓPEZ-FERNÁNDEZ, M. \& ROMERO-FERNÁNDEZ, P.M. (2017): “Towards a configuration of socially responsible human resource management policies and practices: findings from an academic consensus", International Journal of Human Resource Management, pp. 1-37. https://doi.org/10.1080/09585192.2017.1332669.

BASTERRETXEA, I. \& ALBIZU, E. (2010): “¿Es posible resistir a la crisis?: Un análisis desde la gestión de las políticas de formación y empleo en Mondragón", CIRIEC-España Revista de Economía Pública, Social y Cooperativa, 67, 75-96.

BHATTACHARYA, M., GIBSON, D.E. \& DOTY, D.H. (2005): "The effects of flexibility in employee skills, employee behaviors, and human resource practices on firm performance", Journal of Management, 31(4), 622-640. https://doi.org/10.1177/0149206304272347.

BORGAZA, C., DEPREDI, S. \& GALERA, G. (2012): "Interpreting social enterprises", Revista de Administração, 47(3), 398-409. https://doi.org/10.5700/rausp1046.

BREAUGH, J.A. (2008): "Employee recruitment: Current knowledge and important areas for future research", Human Resource Management Review, 18(3), 103-118. https://doi.org/10.1016/J.HRMR.2008.07.003.

BUSINESS ROUNDTABLE AN ASSOCIATION (2019): "Business Roundtable "Statement on the Purpose of a Corporation", Proposes New Paradigm. Retrieved from www.sullcrom.com

BUSTAMANTE SALAZAR, A.M. (2019): "Gestión humana socialmente responsable en cooperativas de trabajo asociado colombianas", CIRIEC-España, Revista de Economía Pública, Social y Cooperativa, 95, 217. https://doi.org/10.7203/ciriec-e.95.10433. 
CARDOSO, L., MEIRELES, A. \& PERALTA, C.F. (2012): "Knowledge management and its critical factors in social economy organisations", Journal of Knowledge Management, 16(2), 267-284. https://doi.org/10.1108/13673271211218861.

CEPES - CONFEDERACIÓN EMPRESARIAL ESPAÑOLA DE LA ECONOMÍA SOCIAL (2018): “Empresas más relevantes de la Economía Social 2016-2017". Retrieved from http://www.serconsulting.es/noticias/empresas-mas-relevantes-de-la-economia-social-2016-2017/

CHAVES, R. \& SAJARDO-MORENO, A. (2004): "Social Economy Managers: Between Values and Entrenchment", Annals of Public and Cooperative Economics, 75(1), 139-161. https://doi.org/10.1111/j.1467-8292.2004.00246.x.

COCETA (2018): Informe de Gestión 2018, Confederación Española de Cooperativas de Trabajo Asociado. Retrieved from www.coceta.coop

CONNELLY, S., MARKEY, S. \& ROSELAND, M. (2011): "Bridging sustainability and the social economy: Achieving community transformation through local food initiatives", Critical Social Policy, 31(2), 308-324. https://doi.org/10.1177/0261018310396040.

CONNER, K.R. \& PRAHALAD, C.K. (1996): “A Resource-based Theory of the Firm: Knowledge Versus Opportunism", Organization Science, 7(5), 477-501. https://doi.org/10.1287/orsc.7.5.477.

DACHEUX, E. \& GOUJON, D. (2011): "The solidarity economy: An alternative development strategy?", International Social Science Journal, 62(203-204), 205-215.

https://doi.org/10.1111/j.1468-2451.2011.01804.x.

DE PRINS, P., BEIRENDONCK, L. VAN, DE VOS, A. \& SEGERS, J. (2014): "Sustainable HRM: Bridging theory and practice through the 'Respect Openness Continuity (ROC)'-model", Management Revue, 25(4), 263-284. https://doi.org/10.1688/mrev-2014-04-Prins

DEFOURNY, J. \& NYSSENS, M. (2012): "El enfoque EMES de la empresa social desde una perspectiva comparada", CIRIEC-España, Revista de Economía Pública, Social y Cooperativa, 75, 6-34. Retrieved from https://www.academia.edu/3060108/El_enfoque_EMES_de_empresa_ social_desde_una_perspectiva_comparada.

DELERY, J.E. \& DOTY, D.H. (1996): "Modes of theorizing in strategic human resource management: Tests of universalistic, contingency, and configurational performance predictions", Academy of Management Journal, 39(4), 802-835. https://doi.org/10.2307/256713

DISTEFANO, J.J. \& MAZNEVSKI, M.L. (2000): "Creating value with diverse teams in global management", Organizational Dynamics, 29(1), 45-63. https://doi.org/10.1016/S0090-2616(00)00012-7 
DOCHERTY, P., FORSLIN, J. \& SHANI, A. (2002): “Creating sustainable work systems : emerging perspectives and practice", Saltsa - Joint programme for working life research in Europe. Routledge.

EHNERT, I. (2009): "Sustainable Human Resource Management", Heidelberg: Physica-Verlag HD. https://doi.org/10.1007/978-3-7908-2188-8

EHRGOTT, M., REIMANN, F., KAUFMANN, L. \& CARTER, C.R. (2011): "Social Sustainability in Selecting Emerging Economy Suppliers", Journal of Business Ethics, 98(1), 99-119. https://doi.org/10.1007/s10551-010-0537-7.

EISENBERGER, R., HUNTINGTON, R., HUTCHISON, S. \& SOWA, D. (1986): “Perceived Organizational Support", Journal of Applied Psychology, 71(3), 500-507.

https://doi.org/10.1037/0021-9010.71.3.500.

ELKINGTON, J. (1994): "Towards the Sustainable Corporation: Win-Win-Win Business Strategies for Sustainable Development", California Management Review, 36(2), 90-100.

https://doi.org/10.2307/41165746.

ESTAPÉ DUBREUIL, G. \& TORREGUITART MIRADA, M.C. (2014): "Social Financing Alternatives for Social Economy Enterprises: Coop57", CIRIEC-España, Revista de Economía Pública, Social y Cooperativa, 80, 136-159. Retrieved from www.ciriec.es - www.ciriec-revistaeconomia.es.

FENWICK, T. \& BIEREMA, L. (2008): “Corporate social responsibility: issues for human resource development professionals", International Journal of Training and Development, 12(1), 24-35. https://doi.org/10.1111/j.1468-2419.2007.00293.x.

FLOREA, L., CHEUNG, Y.H. \& HERNDON, N.C. (2013): "For All Good Reasons: Role of Values in Organizational Sustainability", Journal of Business Ethics, 114(3), 393-408.

https://doi.org/10.1007/s10551-012-1355-x.

GEORGE, J.M. \& JONES, G.R. (1997): “Experiencing work: Values, attitudes, and modos”, Human Relations, 50(4), 393-416. https://doi.org/10.1023/A:1016954827770.

GILL, F. (2012): "Practicing environmental responsibility: Local and global dimensions", Social Responsibility Journal, 8(1), 21-32. https://doi.org/10.1108/17471111211196548.

GREENHAUS, J.H., CALLANAN, G.A. \& GODSHALK, V.M. (2010): Career management, SAGE Publications. Retrieved from https://books.google.es/books?id=FQoPkp7_PzIC\&lpg.

GREENING, D.W. \& TURBAN, D.B. (2000): "Corporate Social Performance as a Competitive Advantage in Attracting a Quality Workforce", Business \& Society, 39(3), 254-280.

https://doi.org/10.1177/000765030003900302 
GUILLÉN, C. \& CESPEDES-LORENTE, J. (2003): “Estrategia de negocio y prácticas de recursos humanos en las cooperativas", CIRIEC-España, Revista de Economía Pública, Social y Cooperativa, 63-94.

HUISKAMP, R. \& KLUYTMANS, F. (2004): “Between Employment Relationships and Market Relationships: Dilemmas for HR Management", Management Revue. The International Review of Management Studies, 15(3), 381-398. https://doi.org/10.5771/0935-9915-2004-3-381.

HUSELID, M.A. (1995): "The Impact Of Human Resource Management Practices On Turnover, Productivity, And Corporate Financial Performance", Academy of Management Journal, 38(3), 635-672. https://doi.org/10.5465/256741.

SCHRAMM, J. (2011): "Promoting Sustainability", HR Magazine. Retrieved from https://www.shrm.org/hr-today/news/hr-magazine/Pages/0311focus.aspx.

JACKSON, K. (2012): "An essay on sustainable work systems: Shaping an agenda for future research : An essay on sustainable work systems: Shaping an agenda for future research", Management Revue, 23(3), 1861-9916. https://doi.org/10.1688/1861-9908_mrev_2012_03_Jackson.

JACKSON, K.T. (2016): "Economy of Mutuality: Merging Financial and Social Sustainability", Journal of Business Ethics, 133(3), 499-517. https://doi.org/10.1007/s10551-014-2408-0.

JÄRLSTRÖM, M., SARU, E. \& VANHALA, S. (2018): "Sustainable Human Resource Management with Salience of Stakeholders: A Top Management Perspective", Journal of Business Ethics, 152(3), 703-724. https://doi.org/10.1007/s10551-016-3310-8.

KAMOCHE, K. (1996): "Strategic human resource management within a resource-capability view of the firm", Journal of Management Studies, 33(2), 213-233.

https://doi.org/10.1111/j.1467-6486.1996.tb00158.x.

KAPTEIN, M. \& WEMPE, J.F. (2002): "The Corporation as Moral Entity". In: The Balanced Company. Retrieved from https://papers.ssrn.com/sol3/papers.cfm?abstract_id=2603357.

KARNANI, A. (2011): "Social Entrepreneurship: Beyond the Hype", Innovations: Technology, Governance, Globalization, 6(2), 99-116. https://doi.org/10.1162/inov_a_00072.

KETOLA, T. (2008): "A holistic corporate responsibility model: Integrating values, discourses and actions", Journal of Business Ethics, 80(3), 419-435.

https://doi.org/10.1007/s10551-007-9428-y.

LEE, J. \& PECCEI, R. (2007): "Perceived organizational support and affective commitment: The mediating role of organization-based self-esteem in the context of job insecurity", Journal of Organizational Behavior, 28(6), 661-685. https://doi.org/10.1002/job.431. 
LEY 5/2011, DE 29 DE MARZO, DE ECONOMÍA SOCIAL (2011): Retrieved from https://www.boe.es/boe/dias/2011/03/30/pdfs/BOE-A-2011-5708.pdf.

LIGER, Q., STEFAN, M. \& BRITTON, J. (2016): Social Economy, European Parliament. Directorate General for Internal Policies. Policy Department A: Economic and Scientific Policy. Retrieved from: http://www.europarl.europa.eu/RegData/etudes/STUD/2016/578969/IPOL_ STU\%282016\%29578969_EN.pdf.

LONGINOS MARÍN RIVES, J., ARCAS LARIO, N., MARTÍNEZ LEÓN, I.M. \& OLMEDO CIFUENTES, I. (2012): "Transparency, corporate citizenship and participation as determinats to implement a code of conduct in social economy organisations", REVESCO, Revista de Estudios Cooperativos, 108. https://doi.org/10.5209/rev-REVE.2012.v18.39588.

MAEL, F. \& ASHFORTH, B. (1992): "Alumni and Their Alma Mater: A Partial Test of the Reformulated Model of Organizational Identification", Journal of Organizational Behavior, 13, 103123. https://doi.org/10.1002/job.4030130202.

MARIAPPANADAR, S. (2014): "Stakeholder harm index: A framework to review work intensification from the critical HRM perspective", Human Resource Management Review, 24(4), 313329. https://doi.org/10.1016/j.hrmr.2014.03.009.

MARTÍN ALCÁZAR, F., MIGUEL ROMERO FERNÁNDEZ, P. \& SÁNCHEZ GARDEY, G. (2013): "Workforce diversity in strategic human resource management models", Cross Cultural Management: An International Journal, 20(1), 39-49. https://doi.org/10.1108/13527601311296247.

MAYO, A. \& GISPERT RAMIS, A. (2000): Las organizaciones que aprenden, Gestión 2000.

MELÉ, D. (2005): "Ethical education in accounting: Integrating rules, values and virtues", Journal of Business Ethics, 57(1), 97-109. https://doi.org/10.1007/s10551-004-3829-y.

MELÉ, D. (2012): “Management ethics: placing ethics at the core of good management", Palgrave Macmillan.

MONE, E.M. (2018): Employee Engagement Through Effective Performance Management: a Practical Guide for Managers, Routledge.

NAPATHORN, C. (2018): "How do social enterprises recruit workers? The case of social enterprises in Thailand", Journal of Asia Business Studies, 12(4), 508-532.

https://doi.org/10.1108/JABS-02-2017-0019.

PERKINS, S.J. (2003): "Globalisation and IHRM: Partners in comparative perspective?",Journal of European Industrial Training, 27(9), 461-472.https://doi.org/10.1108/03090590310506478.

PFEFFER, J. (2005): “Changing mental models: HR's most important task", Human Resource Management, 44(2), 123-128. https://doi.org/10.1002/hrm.20053. 
PLESS, N.M. \& MAAK, T. (2004): "Building an inclusive diversity culture: Principles, processes and practice", Journal of Business Ethics, https://doi.org/10.1007/s10551-004-9465-8.

ROCHA, C.M. (2015): "Perceived organizational support and employee performance: The mediating role of organizational commitment and organizational citizenship behavior". Retrieved from https://www.researchgate.net/publication/242076585.

ROGERS, K.M. \& ASHFORTH, B.E. (2017): "Respect in Organizations: Feeling Valued as 'We' and 'Me'”,JournalofManagement,43(5),1578-1608.https://doi.org/10.1177/0149206314557159.

SCHULER, R. \& JACKSON, S.E. (2014): "Human resource management and organizational effectiveness: yesterday and today", Journal of Organizational Effectiveness, 1(1), 35-55. https://doi.org/10.1108/J0EPP-01-2014-0003.

SENGUPTA, S., SAHAY, A. \& CROCE, F. (2018): "Conceptualizing social entrepreneurship in the context of emerging economies: an integrative review of past research from BRIICS", International Entrepreneurship and Management Journal, 14(4), 771-803.

https://doi.org/10.1007/s11365-017-0483-2.

SHARMA, A. \& BHATNAGAR, J. (2016): "Enterprise social media at work: web-based solutions for employee engagement", Human Resource Management International Digest, 24(7), 16-19. https://doi.org/10.1108/HRMID-04-2016-0055.

SMITH, W., GONIN, M. \& BESHAROV, M. (2013): "Managing Social-Business Tensions: A Review and Research Agenda for Social Enterprise", Business Ethics Quarterly, 23, 407-442. https://doi.org/10.5465/AMBPP.2013.187.

SOCIAL ECONOMY EUROPE (2015): "La Economía Social... retoma la iniciativa. Propuestas para hacer de la Economía Social un pilar de la Unión Europea". Retrieved from https://www.economiasolidaria.org/biblioteca/libro-blanco-de-la-economia-social.

STEINER, A. (2018): "The Extraordinary Opportunity of the 2030 Agenda for Sustainable Development", The European Journal of Development Research, 30. https://doi.org/10.1057/s41287-018-0131-x.

STONE, D.L., STONE-ROMERO, E.F. \& LUKASZEWSKI, K.M. (2007): "The impact of cultural values on the acceptance and effectiveness of human resource management policies and practices", Human Resource Management Review, 17(2), 152-165.

https://doi.org/10.1016/j.hrmr.2007.04.003.

SUNLEY, P. \& PINCH, S. (2012): "Financing social enterprise: social bricolage or evolutionary entrepreneurialism?", Social Enterprise Journal, 8(2), 108-122.

https://doi.org/10.1108/17508611211252837. 
TABIU, A. \& NURA, A.A. (2013): "Assessing the Effects of Human Resource Management (HRM) Practices on Employee Job Performance", Journal of Business Studies Quarterly, 5(2), 247-259. Retrieved from https://search.proquest.com/abicomplete/docview/1503664156/E3527A9B2D854CADPQ/7? accountid=14695.

THE WORLD ECONOMIC FORUM (2018): "The Future of Jobs Report 2018 Insight Report Centre for the New Economy and Society". Retrieved from http://www3.weforum.org/docs/WEF_Future_of_Jobs_2018.pdf.

TSUI, A.S. \& WU, J.B. (2005): "The new employment relationship versus the mutual investment approach: Implications for human resource management", Human Resource Management, 44(2), 115-121. https://doi.org/10.1002/hrm.20052.

WAGNER, M. (2013): “'Green” Human Resource Benefits: Do they Matter as Determinants of Environmental Management System Implementation?", Journal of Business Ethics, 114(3), 443456. https://doi.org/10.1007/s10551-012-1356-9.

ZHANG, L., ZHANG, Y., DALLAS, M., XU, S. \& HU, J. (2018): "How perceived empowerment HR practices influence work engagement in social enterprises-a moderated mediation model", International Journal of Human Resource Management, 29(20), 2971-2999.

https://doi.org/10.1080/09585192.2018.1479874. 